\title{
INFILTRACIÓN Y GRUPOS HIDROLÓGICOS DE SUELOS EN LAS LADERAS DE LOS PÁRAMOS (VALLADOLID)
}

\author{
J. MONGIL ${ }^{(1) *}$, J. NAVARRO ${ }^{(2)}$ \\ ${ }^{1}$ Grupo de Hidrología y Conservación. Universidad Católica de Ávila. \\ Canteros s/n 05005-Ávila (España). \\ ${ }^{2}$ Unidad de Hidráulica e Hidrología. Universidad de Valladolid. \\ Av. Madrid 44 34004-Palencia (España).
}

\begin{abstract}
RESUMEN. Se muestran los resultados de tres ensayos de infiltración realizados en otros tantos suelos de la provincia de Valladolid, concretamente en las cuestas de los páramos calizos. Son suelos entre francos y arcillosos, formados en los tres casos sobre el mismo material geológico, en concreto sobre limos y arcillas grises. La vegetación desarrollada sobre estos suelos es en uno de los casos un pinar de Pinus halepensis de repoblación y en los otros dos matorrales en estado desigual de degradación. Con los datos de los ensayos se ha procedido a su ajuste al modelo de Horton y se ha determinado el grupo de suelo según el modelo del Número de Curva. En general, los suelos tienen condiciones de infiltración regulares, aunque mucho mejores en el pinar que en los matorrales, con capacidades finales de infiltración medias-bajas. Según la textura, la conductividad hidráulica a saturación y la tasa de infiltración mínima, los suelos son de tipo $C$ y $D$, con una tendencia hacia el grupo B en el bosque. Estos resultados son de interés para asignar con mejor criterio los Grupos Hidrológicos de Suelos propuestos en el método del Número de Curva, que se usan en los modelos de simulación hidrológicos para la estimación de avenidas y la ordenación del territorio, en torno a las llanuras de inundación, o para estimar los caudales de diseño de las infraestructuras fluviales en cuencas con terrenos similares a los estudiados.
\end{abstract}

Infiltration and soil hydrological groups on the slopes of the limestone moors (Valladolid, Spain)

ABSTRACT. We show the results of three soil infiltration tests that were made in the slopes under the typical limestone moors of the Valladolid Province (Spain). Soils tested have evolved from Tertiary grey silts and clays generating clayey to loamy textures. Vegetation on these soils is a Pinus halepensis forest from reforestation and two Mediterranean brush degraded covers. Horton infiltration model has been adjusted using data from the trials. Soil hydrologic groups from runoff Curve Number model have been also determined for every 
soil tested. Finally, soil infiltration conditions are moderate for everyone, but much better in the pine forest than in the others. Final infiltration rate is mediumto-low. Soils belong to $C$ and D Hydrologic Soil Groups according to their texture, saturated hydraulic conductivity and minimum infiltration rate, although pine forest shows a certain group B quality. These results would allow better approach for the Hydrologic Soil Groups proposed by the Curve Number Method for using them in simulation hydrologic models to estimate floods and land-use planning or to estimate design flows of fluvial infrastructures in river basins with similar lands to those studied here.

Palabras clave: conductividad hidráulica saturada, escorrentía, Número de Curva, Horton, suelos forestales.

Key words: saturated hydraulic conductivity, runoff, SCS Curve Number, Horton, forest soils.

Enviado el 27 de julio de 2011

Aceptado el 26 de enero de 2012

*Correspondencia: Grupo de Hidrología y Conservación. Universidad Católica de Ávila. Canteros s/n 05005-Ávila (España).E-mail: jorge.mongil@ucavila.es

\section{Introducción}

La infiltración es un proceso hidrológico complejo que consiste en la entrada de agua en el suelo a través de su superficie, que se debe a fuerzas gravitacionales y capilares (Martínez de Azagra y Navarro, 1996). Se trata de uno de los principales componentes del ciclo hidrológico, y un auténtico motor de las relaciones ecológicas necesarias para el funcionamiento de los ecosistemas terrestres dependientes del agua (Martínez de Azagra et al., 2006a; Martínez de Azagra et al., 2006b). Es este un proceso muy estudiado y que ha sido objeto de numerosos intentos de modelización (por ejemplo: Green y Ampt; 1911; Philip, 1957; Holtan, 1961; Overton, 1964; Fitzgerald et al., 1971; Collis-George, 1977; Rawls y Brakensiek, 1983; Al-Azawi, 1985; Rawls et al.; 1993; Ravi y Williams, 1998; Williams et al., 1998; Martínez de Azagra et al., 2006a). Sin embargo, debido a que depende de un gran número de factores, su modelización no es sencilla. Estos factores son (Skaggs y Khaheel, 1982; Cerdà, 1995; Martínez de Azagra y Navarro, 1996), principalmente, la precipitación (intensidad, duración y tamaño de las gotas), características del agua que ha de infiltrarse, la topografía del terreno, la cubierta vegetal, el suelo (estado de su superficie, profundidad, textura, elementos gruesos, estructura, porosidad y densidad, contenido de agua, materia orgánica, presencia de arcilla y carbonato cálcico, entre otras características), y tratamientos realizados en el suelo tales como labores agroforestales. La tasa de infiltración es la cantidad de agua que se infiltra a través de la superficie del suelo en un determinado instante $\left(\mathrm{mm} \cdot \mathrm{h}^{-1}\right)$. Asimismo, la capacidad de infiltración es la cantidad máxima de agua que podría infiltrarse en un suelo por unidad de tiempo y en un instante determinado. Tasa y capacidad de infiltración son términos que en ocasiones se utilizan 
indistintamente, pero no son exactamente lo mismo, ya que si la intensidad de lluvia es menor que la capacidad de infiltración, la tasa de infiltración no coincide con la capacidad; es decir, hay una infinita variedad de tasas pero sólo una capacidad de infiltración para un suelo concreto y en un momento determinado (Horton, 1940).

A la dificultad de modelización mencionada anteriormente, se une el problema de realizar mediciones fiables, ya que la medición de la tasa de infiltración es muy dependiente del método utilizado (Cerdà, 1995; Touma y Albergel, 1992; Blanco, 1999). Por todo ello, y a pesar de las numerosas publicaciones sobre el tema, el conocimiento de este componente del ciclo hidrológico no es aún óptimo. Esto es más patente en terrenos forestales, en los que, no obstante, se han llevado a cabo numerosas experiencias como las de Molina (1993), Madrid et al. (2006), Buczko et al. (2006), Bens et al. (2007), Ilstedt et al. (2007), Zapata y Manzano (2008), Yaseef et al. (2008), Valeron y Meixner (2010), Bonell et al. (2010), Huang et al. (2010) y Alaoui et al. (2011), entre otros. Todos estos trabajos han sido realizados en condiciones ambientales notablemente diferentes a las del presente estudio y ponen de manifiesto el elevado número de factores que intervienen en la infiltración.

El objetivo de este trabajo es establecer una aproximación para el conocimiento del proceso de infiltración en laderas de la zona central de la cuenca del Duero, concretamente en dos parcelas con matorral y otra más de pinar de Pinus halepensis Mill. procedente de repoblación, mediante ensayos con infiltrómetro inundador y ajuste al modelo de infiltración de Horton (1940). El interés de conocer mejor la infiltración en estas laderas radica en que son elementos geomorfológicos típicos de esta zona de la cuenca del Duero, que en muchos casos sufren procesos de erosión y desertificación por aridez edáfica, y en otros han sido objeto de restauración forestal. Por otra parte, existe una cierta escasez de estudios sobre infiltración en la cuenca del Duero, en relación a otras zonas semiáridas de España (Ceballos et al., 2002). Así mismo se pretende estimar el Grupo Hidrológico de Suelo del método del Número de Curva (SCS, 1991), método profusamente empleado para deducir la escorrentía de un aguacero en ingeniería hidrológica, impacto ambiental y planificación del territorio, por su relativa sencillez. Sin embargo, a pesar de su uso frecuente a nivel internacional, fuera de Estados Unidos se hallan pobremente definidos los grupos hidrológicos de suelos y se carece de protocolos e instrucciones específicas para determinarlos (Hawkins et al., 2009). Este trabajo se realiza con el fin de mejorar la asignación del número de curva de los diferentes complejos hidrológicos, lo que facilitará la estimación de la escorrentía superficial en estudios hidrológicos que pudieran llevarse a cabo en la zona.

\section{Material y métodos}

\subsection{Zona de estudio}

Los muestreos se han realizado en tres puntos, dos de ellos situados en el municipio de Geria y el tercero en el término municipal de Matilla de los Caños. En la tabla 1 se muestran las principales características de los puntos de muestreo. Estos dos munici- 


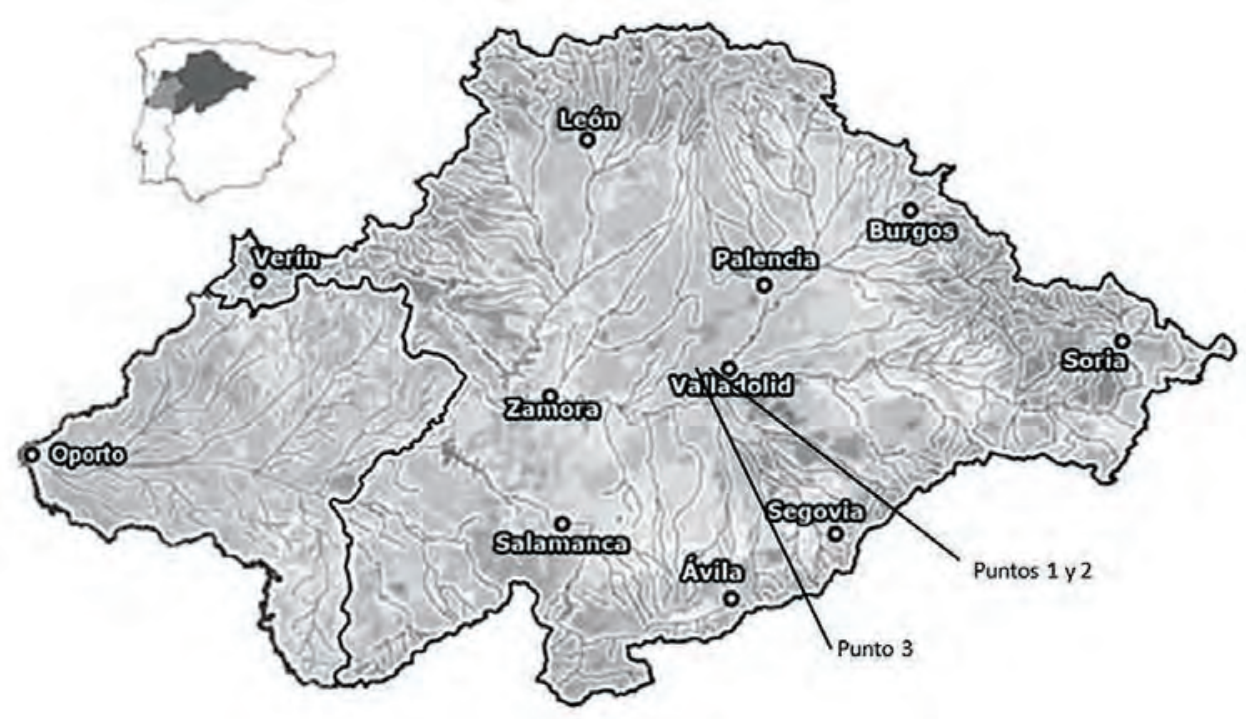

Figura 1. Localización de los puntos de muestreo en la cuenca del Duero.

pios pertenecen a la provincia de Valladolid (España), y se localizan a una distancia de la capital de $15 \mathrm{~km}$ en el caso de Geria y $23 \mathrm{~km}$ en el caso de Matilla. Están situados muy próximos entre sí, en la parte central de la cuenca del Duero, unos pocos kilómetros al norte de este río (fig. 1).

Tabla 1. Caracterización de los puntos de muestreo.

\begin{tabular}{|c|c|c|c|c|c|c|}
\hline PM & LOCALIDAD & COORD & H & EX & PT & VEGETACIÓN \\
\hline 1 & Geria & $342766 ; 4606484$ & 835 & S & 24 & $\begin{array}{c}\text { Pinar de Pinus halepensis } \\
\text { de repoblación. Fcc }=0,9\end{array}$ \\
\hline 2 & Geria & $343426 ; 4606236$ & 780 & S & 33 & $\begin{array}{c}\text { Matorral de Dorycnium } \\
\text { pentaphylum y } \text { Rosa } \text { sp., } \\
\text { entre otras especies. Fcc }=0,8\end{array}$ \\
\hline 3 & Matilla de los Caños & $336536 ; 4602515$ & 778 & S-SE & 26 & $\begin{array}{c}\text { Matas leñosas y herbáceas } \\
\text { de diversas especies. Fcc }=0,7\end{array}$ \\
\hline
\end{tabular}

PM: Punto de muestreo; COORD: Coordenadas UTM (huso 30T, datum WGS84); H: Altitud (m); EX: Exposición de la ladera; PT: Pendiente (\%); Fcc: Fracción de cabida cubierta.

El clima de la zona es mediterráneo continental, con una precipitación anual media de $451 \mathrm{~mm}$ y una temperatura media anual de $12.1^{\circ} \mathrm{C}$. Los suelos (tabla 2) son Regosoles Calcáreos (FAO, 2006), con un perfil AC, siendo el horizonte A de unos pocos centímetros y algo más oscuro que el horizonte $\mathrm{C}$. Tienen texturas que van de franca a 
arcillosa, con escasa materia orgánica, $\mathrm{pH}$ básico, alto contenido en carbonatos y tendencia al encostramiento con cubierta vegetal degradada. Se han formado en los tres casos sobre los limos y arcillas grises del tramo inferior de la Facies de las Cuestas, de edad Vallesiense (Mioceno superior). En algún caso, especialmente en el punto de muestreo 3, aparecen intercalados niveles de calizas y margocalizas de colores claros y grises.

Tabla 2. Características de los suelos de los diferentes puntos de muestreo.

\begin{tabular}{|c|c|c|c|c|c|c|c|c|c|c|}
\hline PM & RM & EG & AR & L & AC & $\begin{array}{c}\text { TEXTURA } \\
\text { USDA }\end{array}$ & MO & $\mathbf{K}_{\text {sat }}$ & CH & OBS \\
\hline 1 & $\begin{array}{c}\text { Limos y } \\
\text { arcillas } \\
\text { grises }\end{array}$ & 23.25 & 43.0 & 37.6 & 19.4 & Franca & 3.32 & 12.5 & $\begin{array}{c}\text { I (seco), } \\
\text { húmedo } \\
\text { al tacto }\end{array}$ & $\begin{array}{c}\text { Abundante pinocha, } \\
\text { raíces abundantes, } \\
\text { pedregosidad abundante, } \\
\text { musgo en superficie }\end{array}$ \\
\hline 2 & $\begin{array}{c}\text { Limos y } \\
\text { arcillas } \\
\text { grises }\end{array}$ & 19.31 & 27.4 & 44.0 & 28.6 & $\begin{array}{c}\text { Franco } \\
\text { arcillosa }\end{array}$ & 0.59 & 4.4 & I (seco) & $\begin{array}{c}\text { Sin restos vegetales, } \\
\text { sin raíces, }\end{array}$ \\
\hline 3 & $\begin{array}{c}\text { Limos y } \\
\text { arcillas } \\
\text { grises }\end{array}$ & 0.04 & 18.0 & 29.7 & 52.3 & Arcillosa & 2.20 & 0.9 & I (seco) & $\begin{array}{c}\text { Sin restos vegetales, } \\
\text { costras y grietas } \\
\text { superficiales }\end{array}$ \\
algunas raíces, sin \\
pedregosidad, costras \\
y grietas superficiales
\end{tabular}

PM: Punto de muestreo; RM: Roca madre; EG: Elementos gruesos (\%); AR: Arena (\%); L: Limo (\%); AC: Arcilla (\%); MO: Materia orgánica (\%); $\mathrm{K}_{\text {sat }}$ : Conductividad hidráulica a saturación $\left(\mathrm{mm} \cdot \mathrm{h}^{-1}\right)$ según el diagrama de la figura 9; CH: Condición previa de humedad en el momento del ensayo según el modelo del Número de Curva (SCS, 1954); OBS: Observaciones.

Los puntos de muestreo están situados en el dominio geomorfológico de las cuestas, de fuertes pendientes, que son el enlace entre las zonas bajas llanas (campiñas) y las zonas altas también llanas (páramos), unidades geomorfológicas todas ellas características de la parte central de la cuenca del Duero.

El punto de muestreo 1 (fig. 2) está situado bajo la cubierta de un pinar de Pinus halepensis Mill. procedente de una repoblación efectuada en el año 1955 y un sotobosque de matas leñosas y herbáceas de Genista scorpius L., Dorycnium pentaphylum Scop., Ononis fruticosa L. y Linnum suffruticosum L., entre otras especies. La fracción de cabida cubierta del arbolado es del 60\%, y la total del $90 \%$. El punto de muestreo 2 (fig. 3) tiene una cubierta vegetal de matas herbáceas y leñosas, de poca altura, de Dorycnium pentaphylum Scop., Astragalus monspessulanus L., Helianthemun hirtum (L.) Miller, Helicrysum stoechas (L.) Moench, Lavandula latifolia Medik., Santolina chamaecyparissus (L.), Thymus mastichina L. y Rosa sp., entre otras especies, con fracción de cabida cubierta del matorral del 80\%, y algunos síntomas de erosión hídrica (laminar, en regueros y en cárcavas). Finalmente, el punto de muestreo 3 (fig. 4) posee unas algunas matas leñosas y herbáceas de escasa talla (Dorycnium pentaphylum Scop., 


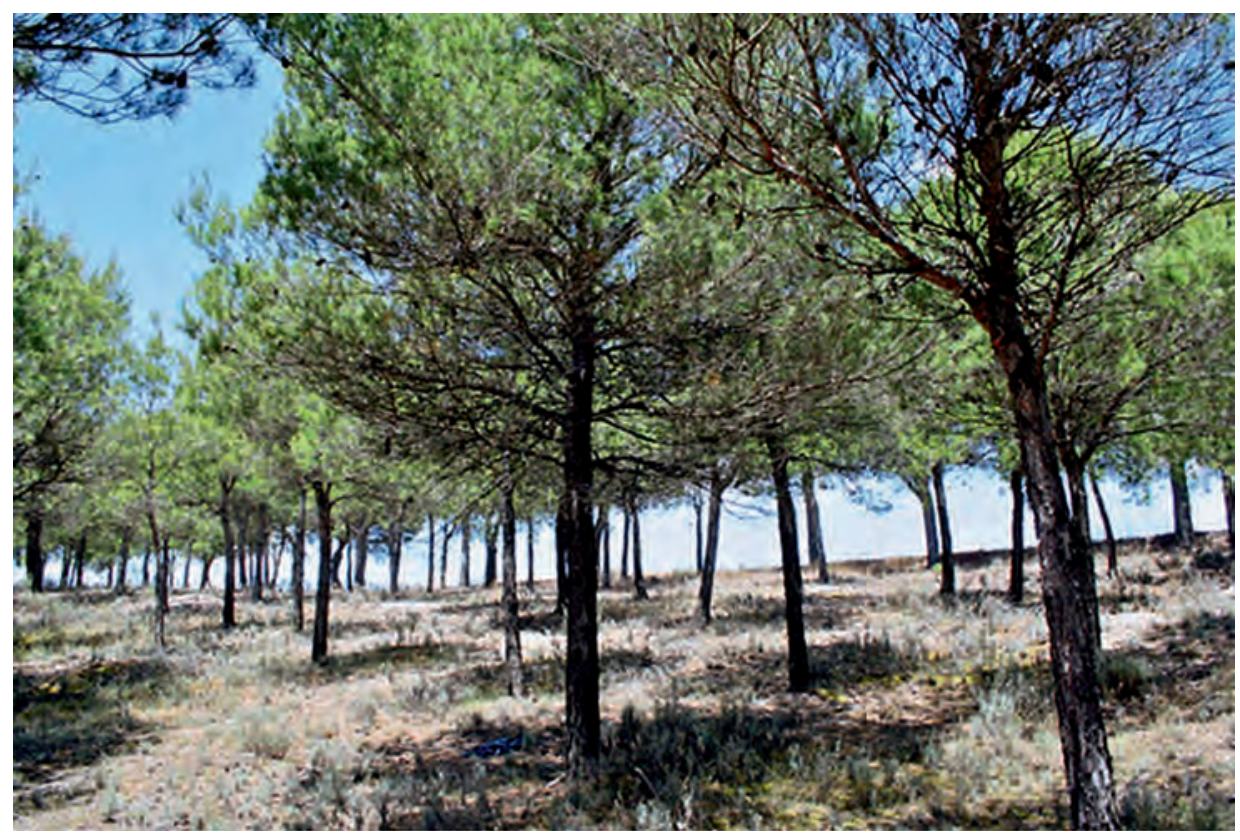

Figura 2. Punto de muestreo 1 (Geria, pinar de Pinus halepensis).

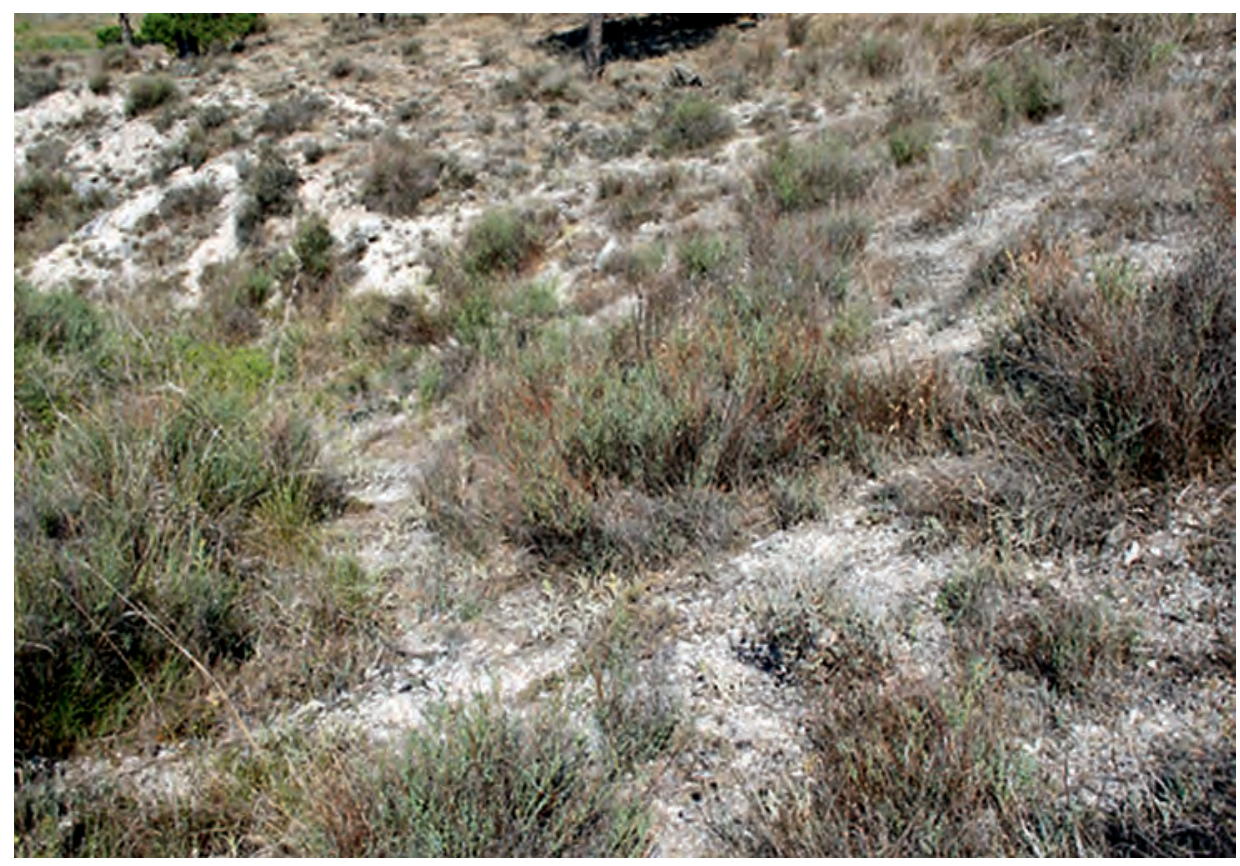

Figura 3. Punto de muestreo 2 (Geria, matorral). 


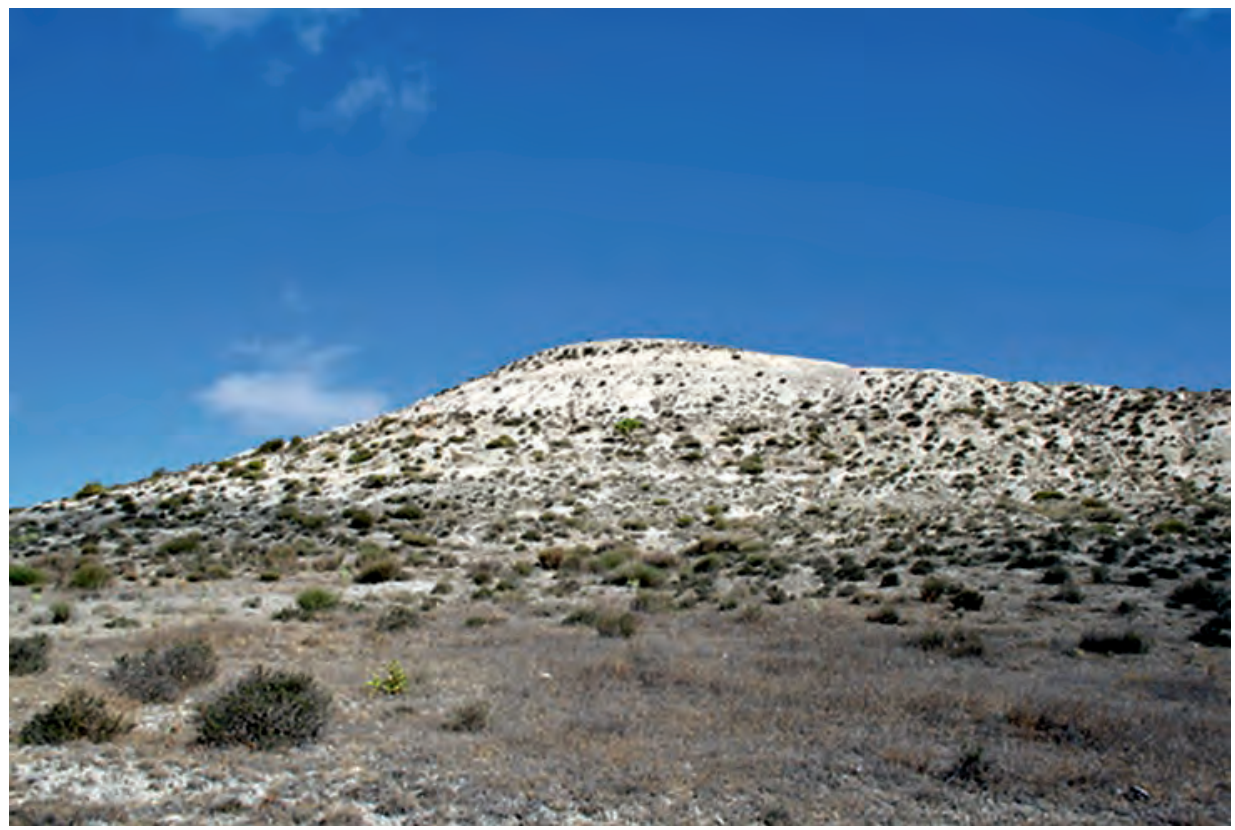

Figura 4. Punto de muestreo 3 (Matilla de los Caños, matorral).

Astragalus monspessulanus L., Santolina chamaecyparissus (L.) y Thymus mastichina L., entre otras), que cubren el suelo en un $70 \%$, con avanzado estado de degradación y signos frecuentes de erosión laminar, en regueros y en cárcavas.

\subsection{Ensayos y mediciones}

Las técnicas de medición de la infiltración son diversas, como indican, por ejemplo, Brakensiek et al. (1979a,b), Hillel (1971, 1980), Haan et al. (1982), Hewlett (1982), Cerdà (1995) y Blanco (1999). De entre los dos métodos más utilizados (Sidiras y Roth, 1987; Cerdà, 1995; etc.), simuladores de lluvia e infiltrómetros inundadores (de uno o varios anillos), se ha elegido el segundo, fundamentalmente por la mayor sencillez en su utilización en campo y por ser recomendable en suelos con encostramientos (Touma y Albergel, 1992). Algunos estudios han obtenido valores de la tasa de infiltración de unas ocho veces más con infiltrómetro inundador que con simulación de lluvia (Cerdà, 1995, 1997), quizás porque los simuladores de lluvia trabajan con intensidades más altas y diferentes velocidades terminales de lluvia que las naturales (Clarke y Walsh, 2007). El infiltrómetro utilizado en este estudio es similar al empleado por Cerdà (1995) en terrenos similares de la vertiente levantina, lo cual facilita luego la comparación y discusión de resultados. Se trata de un cilindro simple de metacrilato de $25 \mathrm{~cm}$ de altura, $8.4 \mathrm{~cm}$ de diámetro interior y $0.3 \mathrm{~cm}$ de grosor de pared (fig. 5). El cilindro de metacrilato es resistente y transparente, por lo que permite conocer posibles incidencias ocurridas 


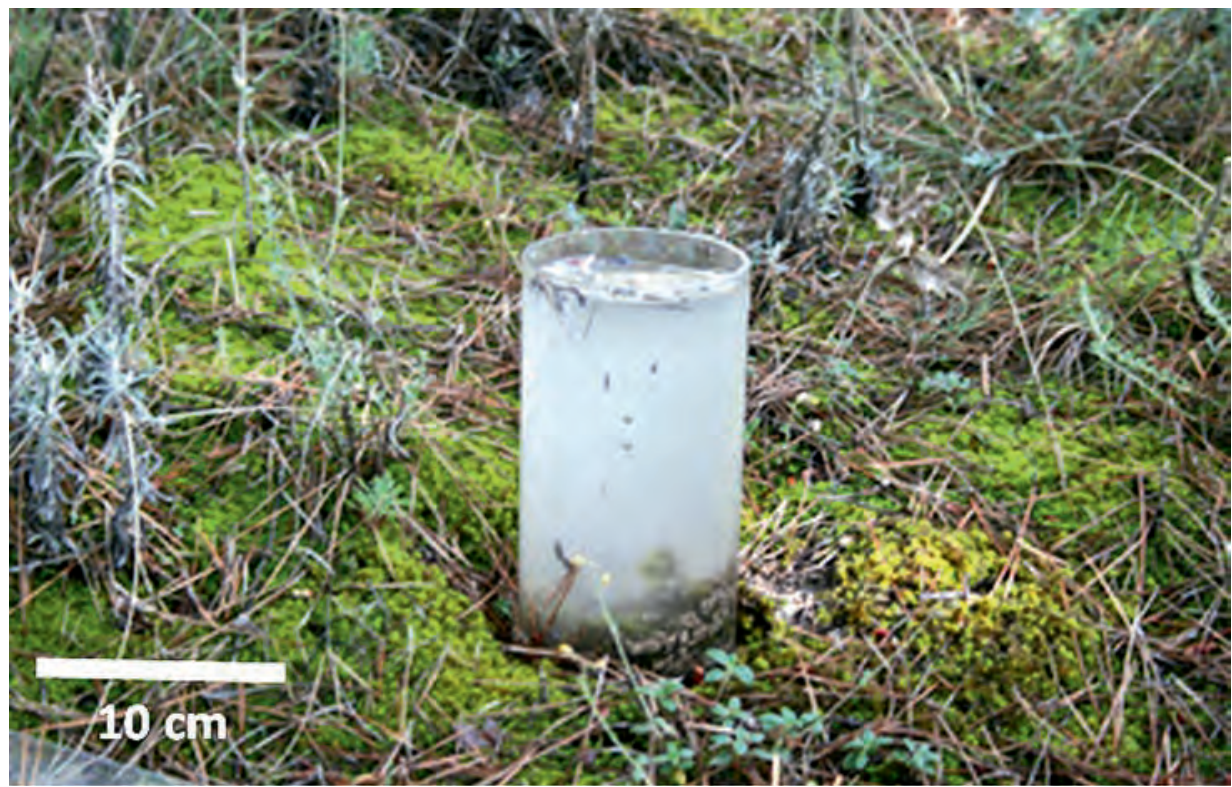

Figura 5. Ensayo de infiltración con el infiltrómetro inundador de metacrilato en el punto de muestreo 1.

durante el experimento y medir la altura de agua con mayor precisión. El ensayo consiste en rellenar con agua el cilindro (encharcamiento o inundación) y medir la altura de agua infiltrada en intervalos de tiempo. Tras cada medición se rellena el cilindro y se humedece la zona colindante con el fin de reducir el flujo lateral, como aconseja, por ejemplo, Harvey (1982).

Después de realizar los ensayos, se elaboraron las gráficas de tasa de infiltración desde el comienzo del ensayo y en cada intervalo, y de altura infiltrada. A continuación se ajustaron los datos de tasa de infiltración al modelo de Horton y se realizó la asignación de los suelos a los diferentes grupos hidrológicos de suelos según su condición para la infiltración, estimada a partir de la textura, la capacidad de infiltración y la conductividad hidráulica saturada.

\section{Resultados y discusión}

\subsection{Ensayos de infiltración}

En la tabla 3 y en las figs. 6, 7 y 8 se ofrecen los principales resultados de los tres ensayos de infiltración realizados, en concreto los valores de infiltración inicial y final. A la vista de esta tabla y de la tabla 2, se observa una mejora en las condiciones generales del suelo y más concretamente de las condiciones de infiltración, con- 
forme la vegetación es más densa y evolucionada. Así, partiendo de un mismo material geológico (limos y arcillas), el suelo tiene una textura arcillosa en el matorral del punto de muestreo 3 , franco-arcillosa en el matorral del punto 2 y franca en el pinar del punto 1. Paralelamente la conductividad hidráulica saturada, deducida a partir de la granulometría del suelo, varía de 0.9 a $12.5 \mathrm{~mm} \cdot \mathrm{h}^{-1}$, en los diferentes puntos de muestreo.

Tabla 3. Tasa de infiltración inicial $\left(f_{0}\right)$ y final $\left(f_{c}\right)\left(\mathrm{mm} \cdot \mathrm{h}^{-1}\right)$ en los ensayos de infiltración realizados.

\begin{tabular}{|c|c|c|}
\hline $\mathbf{P M}$ & $\begin{array}{c}\mathbf{f}_{\mathbf{0}} \\
\left(\mathbf{m m} \cdot \mathbf{h}^{-\mathbf{1}}\right)\end{array}$ & $\begin{array}{c}\mathbf{f}_{\mathbf{c}} \\
\left(\mathbf{m m} \cdot \mathbf{h}^{-\mathbf{1}}\right)\end{array}$ \\
\hline 1 & 2400 & 184 \\
\hline 2 & 150 & 22 \\
\hline 3 & 90 & 8 \\
\hline
\end{tabular}

PM: Punto de muestreo.

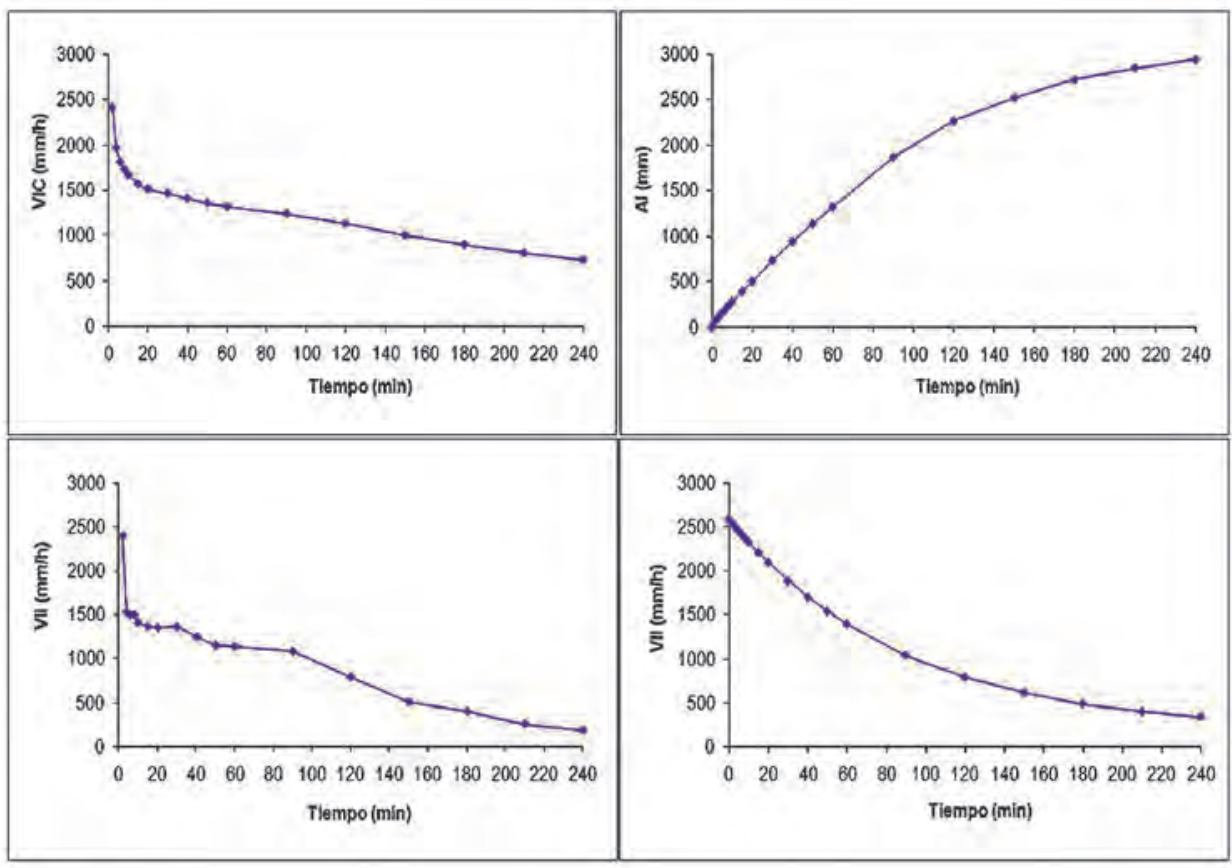

Figura 6. Tasa de infiltración desde el comienzo del ensayo (VIC) $\left(\mathrm{mm} \cdot \mathrm{h}^{-1}\right)$, altura infiltrada (AI) $(\mathrm{mm})$ y tasa de infiltración en cada intervalo (VII) $\left(\mathrm{mm} \cdot \mathrm{h}^{-1}\right)$ (en el ensayo y según el modelo de Horton) para el punto de muestreo 1 (Geria, pinar). 


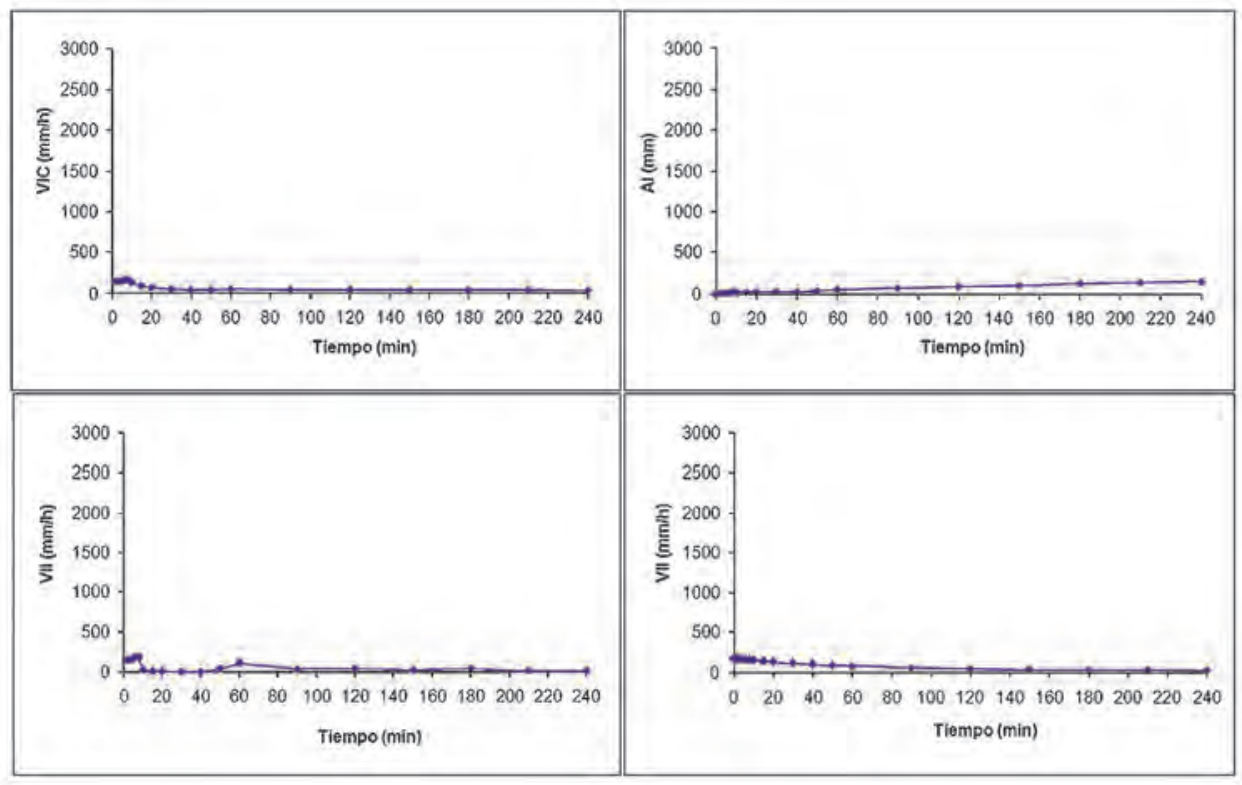

Figura 7. Tasa de infiltración desde el comienzo del ensayo (VIC) $\left(\mathrm{mm} \cdot \mathrm{h}^{-1}\right)$, altura infiltrada (AI) $(\mathrm{mm})$ y tasa de infiltración en cada intervalo (VII) $\left(\mathrm{mm} \cdot \mathrm{h}^{-1}\right)$ (en el ensayo y según el modelo de Horton) para el punto de muestreo 2 (Geria, matorral).

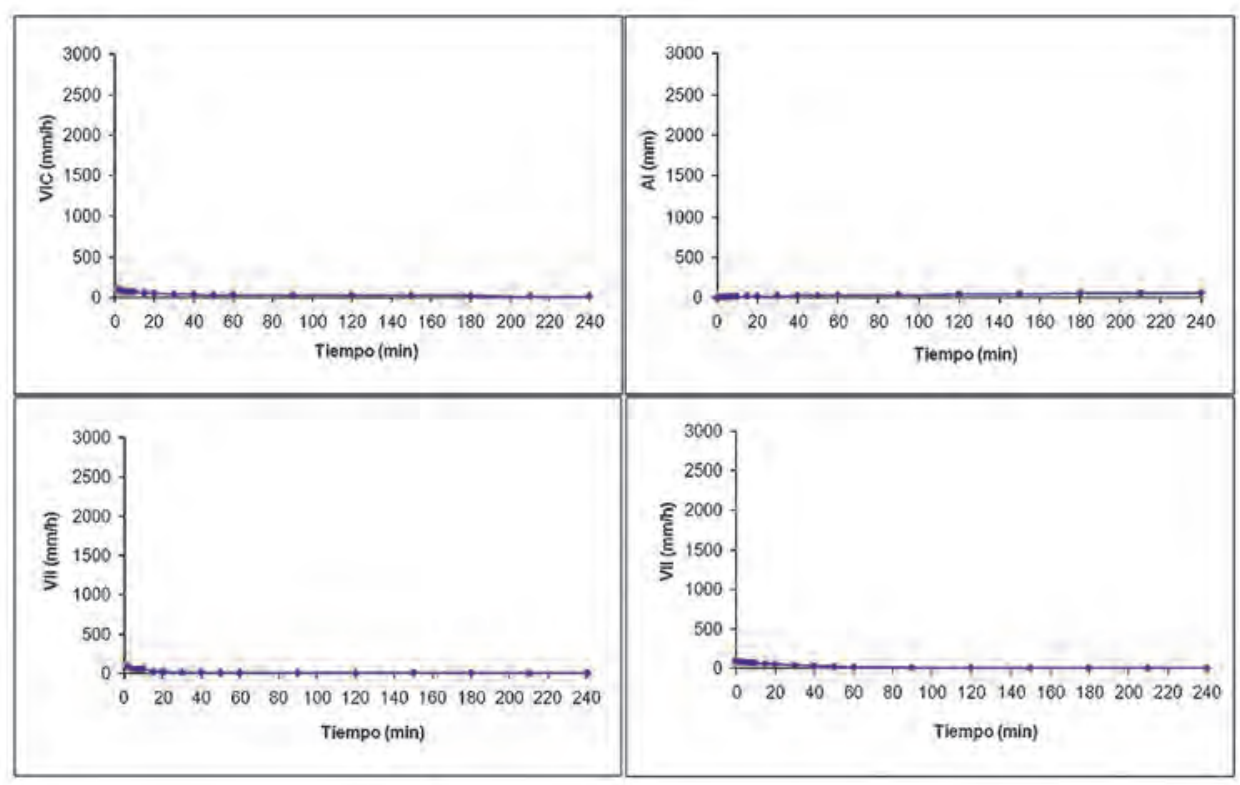

Figura 8. Tasa de infiltración desde el comienzo del ensayo (VIC) $\left(\mathrm{mm} \cdot \mathrm{h}^{-1}\right)$, altura infiltrada (AI) $(\mathrm{mm})$ y tasa de infiltración en cada intervalo (VII) $\left(\mathrm{mm} \cdot \mathrm{h}^{-1}\right)$ (en el ensayo y según el modelo de Horton) para el punto de muestreo 3 (Matilla, matorral). 
Respecto a las tasas de infiltración, hay que decir en primer lugar que los valores tanto iniciales como finales son bastante altos: las tasas iniciales de infiltración obtenidas son 2400,150 y $90 \mathrm{~mm} \cdot \mathrm{h}^{-1}$, respectivamente para el pinar y las dos zonas de matorral; y las tasas de infiltración final son 184.22 y $8 \mathrm{~mm} \cdot \mathrm{h}^{-1}$, para los tres puntos en estudio. No obstante, estos resultados son comparables a los que aportan otros autores en suelos y vegetación relativamente similares. Por ejemplo, Cerdà (1995) obtuvo, mediante infiltrómetro inundador, para suelos diversos incluyendo los formados sobre arcillas o margas, y cubiertas vegetales de matorral, herbáceas o suelos desnudos, valores iniciales de infiltración $\left(\mathrm{f}_{0}\right.$ ) entre 292 y $8190 \mathrm{~mm} \cdot \mathrm{h}^{-1}$ y finales (f $\mathrm{f}_{\mathrm{c}}$ ) entre 30 y 958 $\mathrm{mm} \cdot \mathrm{h}^{-1}$, más elevados que los obtenidos en nuestro caso. También hay que señalar que los infiltrómetros menores a $15 \mathrm{~cm}$ de diámetro pueden mayorar en más de un $30 \%$ las tasas de infiltración (Tricker, 1978), pero son muy manejables en campo, lo cual muchas veces es el mayor condicionante de operación, sobre todo en zonas de fuerte pendiente (Blanco, 1999), como era este caso. Por otro lado, Ceballos et al. (2002) obtienen en texturas arenosas del Duero Central tasas finales mayores de $40 \mathrm{~mm} \cdot \mathrm{h}^{-1}$ en viñedos abandonados y zonas repobladas, empleando simuladores de lluvia, que quedan bastante por encima de nuestros suelos de textura más desfavorable para la infiltración.

En cuanto a las tasas de infiltración inicial y final, son mucho mayores en el pinar que en los matorrales, y entre éstos, el suelo mucho más degradado de Matilla (punto 3) presenta las menores tasas de infiltración. En concreto, el pinar tiene una tasa de infiltración inicial 16 veces mayor que la del matorral del punto de muestreo 2 y 26.7 veces mayor que la del punto de muestreo 3; y la tasa de infiltración final del pinar es, respectivamente, 8.4 y 23 veces mayor que la de los matorrales. Estos resultados son concordantes con los de otros autores: Cerdà (1995) afirma que la cubierta vegetal tiene una clara relación positiva con la infiltración, de tal manera que cuanto más abundante y densa sea la cubierta vegetal mejores resultan las condiciones de infiltración y en términos similares se pronuncian Hewlett (1982) y López Cadenas y Mintegui (1986). No obstante, si analizamos los datos texturales, el bosque es el terreno más arenoso y menos arcilloso, lo que en la diferencia de comportamiento puede influir también. Por otra parte, en los ensayos de Ceballos et al. (2002) en la zona central del Duero, aunque no estudian la relación de la infiltración con la cubierta vegetal, se puede observar que las parcelas de viña y reforestadas presentan mejores tasas de infiltración $\left(>40 \mathrm{~mm} \cdot \mathrm{h}^{-1}\right)$ que los cultivos abandonados y las viñas con pedregosidad superficial $\left(\leq 40 \mathrm{~mm} \cdot \mathrm{h}^{-1}\right)$.

Se han ajustado los datos de los ensayos de infiltración al modelo de Horton, con el fin de obtener ecuaciones que permitan predecir la tasa de infiltración en cualquier momento t. Los resultados del ajuste se muestran en las tablas 4 y 5, y en las figuras 6 a 8 . El modelo de Horton $(1937,1940)$ permite simular la curva de tasa de infiltración de un suelo. Se trata de uno de los modelos empíricos de infiltración más populares (Chang, 2006) y sencillo, y expresa la tasa de infiltración en función del tiempo, de constantes empíricas y de parámetros del suelo. La expresión del modelo es:

$$
f=f(t)=f_{c}+\left(f_{o}-f_{c}\right) \cdot e^{-k . t}
$$


Tabla 4. Resultados del ajuste al modelo de Horton: tasa de infiltración inicial $\left(f_{0}\right)$ y final $\left(f_{c}\right)$ en $\mathrm{mm} \cdot h^{-1}$, y exponente de decrecimiento $(k)$ en $h^{-1}$.

\begin{tabular}{|c|c|c|c|}
\hline $\mathbf{P M}$ & $\begin{array}{c}\mathbf{f}_{\mathbf{0}} \\
\left(\mathbf{m m} \cdot \mathbf{h}^{-\mathbf{1}}\right)\end{array}$ & $\begin{array}{c}\mathbf{f}_{\mathbf{c}} \\
\left(\mathbf{m m} \cdot \mathbf{h}^{-\mathbf{1}}\right)\end{array}$ & $\begin{array}{c}\mathbf{k} \\
\left(\mathbf{h}^{-1}\right)\end{array}$ \\
\hline 1 & 2584 & 338 & 0.01144 \\
\hline 2 & 172 & 25.8 & 0.01527 \\
\hline 3 & 90 & 8 & 0.03172 \\
\hline
\end{tabular}

PM: Punto de muestreo.

Tabla 5. Diferencias de los parámetros del modelo de Horton respecto a los valores obtenidos empíricamente.

\begin{tabular}{|c|c|c|}
\hline PM & $\begin{array}{c}\text { DNf } \\
(\boldsymbol{\%})\end{array}$ & $\begin{array}{c}\text { DNf } \\
(\boldsymbol{\%})\end{array}$ \\
\hline 1 & 7.7 & 83.7 \\
\hline 2 & 14.7 & 17.3 \\
\hline 3 & 0.0 & 0.0 \\
\hline DNM & 7.5 & 33.7 \\
\hline
\end{tabular}

PM: Punto de muestreo

DNf $_{0}$ : Diferencia normalizada de $\mathrm{f}_{0}(\%) \cdot \mathrm{DNf}_{0}=\left(\Delta \mathrm{f}_{0} / \mathrm{f}_{0}\right) \cdot 100$

$D N f_{c}$ : Diferencia normalizada de $f_{c}(\%)$. $D N f_{c}=\left(\Delta f_{c} / f_{c}\right) \cdot 100$

DNM: Diferencia normalizada media (\%)

$\Delta \mathrm{f}_{0}=$ Diferencia entre valores de capacidad de infiltración inicial $\left(\mathrm{mm} \cdot \mathrm{h}^{-1}\right)$

$\Delta \mathrm{f}_{\mathrm{c}}=$ Diferencia entre valores de capacidad de infiltración final $\left(\mathrm{mm} \cdot \mathrm{h}^{-1}\right)$

En esta ecuación, $\mathrm{f}$ es la tasa de infiltración en el tiempo $\mathrm{t}, \mathrm{f}_{0}$ es la tasa de infiltración en un tiempo igual a $0, \mathrm{f}_{\mathrm{c}}$ es la tasa de infiltración constante que se alcanza tras un tiempo $t_{c}$, $y$ k es el exponente de decrecimiento. Los parámetros $\mathrm{f}_{0}, \mathrm{f}_{\mathrm{c}} \mathrm{y} \mathrm{k}$ se deben ajustar mediante datos experimentales procedentes de ensayos de infiltración.

En la tabla 5 se pueden ver las diferencias obtenidas entre las capacidades de infiltración obtenidas en los ensayos y del modelo ajustado de Horton. La diferencia normalizada media es menor para la tasa de infiltración inicial (7.5\%) que para la tasa de infiltración final (33.7\%). En el punto de muestreo 1, para la tasa de infiltración final la diferencia se eleva hasta el $83.7 \%$, lo cual indica que podría ser interesante el ajuste a otros modelos.

\subsection{Determinación del grupo hidrológico de suelo}

El modelo del Número de Curva es un modelo empírico que estima la escorrentía superficial generada por un aguacero, bajo diferentes tipos de suelo y de cubierta vegetal, desarrollado a mediados del siglo XX por el USDA Soil Conservation Service (SCS, 1991) 
y que se emplea con mucha frecuencia, desde entonces, en la modelización para la estimación de caudales en la ingeniería hidrológica, el impacto ambiental y la planificación del territorio (Hawkins et al., 2009). Su aplicación, se basa en establecer los grupos hidrológicos de suelo desde el punto de vista de su comportamiento respecto a la escorrentía, característica que se determina en función de la textura del suelo, la profundidad y su capacidad de infiltración. La clasificación original se debe a Musgrave (1955), y posteriormente se han ido modificando las definiciones y delimitaciones (entre otros: SSDS, 1993; NRCS, 2007). Los cuatro grupos hidrológicos de suelo son (NRCS, 2007):

- Grupo A. Suelos que tienen bajo potencial de escurrimiento aún cuando estén húmedos. Tienen menos del $10 \%$ de arcilla y más del $90 \%$ de arena y/o gravas. Son suelos de texturas arenosas o de gravas, y pueden tener texturas más finas si el suelo presenta una buena estructura de agregados, baja densidad aparente y/o más del 35\% de fragmentos rocosos. En todos los horizontes la conductividad hidráulica a saturación es superior a $144 \mathrm{~mm} \cdot \mathrm{h}^{-1}$, si la capa impermeable se encuentra a más de $50 \mathrm{~cm}$ o el nivel freático se encuentra a más de $60 \mathrm{~cm}$. Suelos con más de $100 \mathrm{~cm}$ de profundidad deben tener una conductividad hidráulica a saturación superior a $36.1 \mathrm{~mm} \cdot \mathrm{h}^{-1}$.

- Grupo B. Se trata de suelos con moderada capacidad de infiltración cuando se encuentran saturados. Poseen de un 10 a un $20 \%$ de arcilla y de un 50 a un $90 \%$ de arena. Tienen texturas franco-arenosa o arenoso-franca, y pueden tener texturas más finas si la estructura presenta buenos agregados, baja densidad aparente y/o más del 35\% de fragmentos rocosos. La conductividad hidráulica a saturación del horizonte más impermeable, en una profundidad hasta $50 \mathrm{~cm}$, está entre 36.1 y $144 \mathrm{~mm} \cdot \mathrm{h}^{-1}$. La profundidad del suelo hasta una capa impermeable es superior a $50 \mathrm{~cm}$ o el nivel freático se encuentra a más de $60 \mathrm{~cm}$. Los suelos de más de $100 \mathrm{~cm}$ de profundidad hasta el nivel freático o hasta la capa impermeable deben tener una conductividad hidráulica a saturación mayor de $14.5 \mathrm{~mm} \cdot \mathrm{h}^{-1}$ en todo el perfil, pero menor de $36.1 \mathrm{~mm} \cdot \mathrm{h}^{-1}$.

- Grupo C. Son suelos de escasa capacidad de infiltración una vez saturados. Tienen de un 20 a un $40 \%$ de arcilla y menos de $50 \%$ de arena. Poseen textura franca, franco-limosa, franco-arcillo-arenosa, franco-arcillosa y franco-arcillo-limosa; o textura arcillosa, arcillo-limosa o arcillo-arenosa, si la estructura presenta buenos agregados, baja densidad aparente y/o más del 35\% de fragmentos rocosos. La conductividad hidráulica a saturación del horizonte más impermeable, hasta $50 \mathrm{~cm}$ de profundidad, está entre 3.6 y $36.1 \mathrm{~mm} \cdot \mathrm{h}^{-1}$. La profundidad a una capa impermeable es mayor de $50 \mathrm{~cm}$ o el nivel freático se encuentra a más de $60 \mathrm{~cm}$. Aquellos suelos con más de $100 \mathrm{~cm}$ de profundidad hasta el nivel freático o hasta la capa impermeable deben tener una conductividad hidráulica a saturación mayor de $1.5 \mathrm{~mm} \cdot \mathrm{h}^{-1}$ en todo el perfil, pero menor de $14.5 \mathrm{~mm} \cdot \mathrm{h}^{-1}$.

- Grupo D. En este grupo los suelos presentan un elevado potencial de escorrentía cuando están completamente saturados de humedad. El movimiento del agua a través del suelo es limitado o muy limitado. Tienen más de un $40 \%$ de arcilla y 
menos de un 50\% de arena. Son suelos de textura arcillosa. Pueden presentar fenómenos de expansión-contracción. Poseen una profundidad menor de $50 \mathrm{~cm}$ a la capa impermeable o menos de $60 \mathrm{~cm}$ al nivel freático. Para suelos con una capa impermeable a una profundidad entre 50 y $100 \mathrm{~cm}$ la conductividad hidráulica a saturación del horizonte más impermeable es inferior a $3.6 \mathrm{~mm} \cdot \mathrm{h}^{-1}$. Suelos que tengan más de $100 \mathrm{~cm}$ de profundidad hasta el nivel freático o la capa impermeable deben tener una conductividad hidráulica a saturación inferior de 1.5 $\mathrm{mm} \cdot \mathrm{h}^{-1}$ en todo el perfil.

Además de estas descripciones, en las que la asignación del tipo de suelo se hace fundamentalmente mediante la textura y la conductividad hidráulica a saturación $\left(\mathrm{K}_{\mathrm{sat}}\right)$, para conocer a qué grupo corresponde un suelo se puede hallar su tasa de infiltración mínima (f $\mathrm{f}_{\mathrm{c}}$ (Hawkins et al., 2009), y acudir a la clasificación de la tabla 6, en la que se han agrupado las clasificaciones más destacadas propuestas hasta ahora. La conductividad hidráulica a saturación $\left(\mathrm{K}_{\mathrm{sat}}\right)$ se obtiene mediante un permeámetro a partir de una muestra inalterada de suelo o, en España, mediante el diagrama propuesto por López Cadenas (1998) de la fig. 9, si se dispone sólo de datos de textura USDA. La obtención de $f_{c}$ se realiza mediante un ensayo de infiltración como el que se ha descrito anteriormente en este trabajo, o mediante un simulador de lluvia (Hawkins et al., 2009). La tasa mínima de infiltración resulta inferior a la conductividad hidráulica por las interferencias que provoca el aire confinado en ciertos poros del suelo, lo que Bouwer (1966) cuantifica mediante la siguiente expresión:

$$
f_{c}=0.5 \cdot K_{\text {sat }}
$$

Tabla 6. Grupos de suelo para el modelo del Número de Curva, según la tasa mínima de infiltración $\left(f_{c}\right)$, la conductividad hidráulica a saturación $\left(K_{\text {sar }}\right)$ y el porcentaje de arena.

\begin{tabular}{|c|c|c|c|c|c|c|}
\hline \multirow[b]{2}{*}{ GRUPO } & \multirow{2}{*}{$\begin{array}{c}\mathbf{f}_{\mathrm{c}}\left(\mathbf{m m} \cdot \mathbf{h}^{-1}\right) \\
\text { Musgrave } \\
(\mathbf{1 9 5 5 )}\end{array}$} & \multicolumn{4}{|c|}{$\mathrm{K}_{\mathrm{sat}}\left(\mathbf{m m} \cdot \mathbf{h}^{-1}\right)$} & \multirow{2}{*}{$\begin{array}{c}\text { \% Arena } \\
\text { Bouwer (1969) (en } \\
\text { Neitsch } \text { et al., } \\
\text { 2002) }\end{array}$} \\
\hline & & $\begin{array}{l}\text { SSDS } \\
\text { (1993) }\end{array}$ & $\begin{array}{l}\text { NRCS } \\
(1993)\end{array}$ & $\begin{array}{c}\text { Nearing } e t \\
\text { al. (1996) }\end{array}$ & $\begin{array}{l}\text { Porta et al. (1999) } \\
\text { y Trueba } \text { et al. } \\
\quad(2000)\end{array}$ & \\
\hline A & $7.62-11.43$ & $>198$ & $\geq 180$ & $\geq 28.36$ & $\geq 50$ & 89-91 \\
\hline B & $3.81-7.62$ & 19.8-198 & $18-180$ & $2.34-16.74$ & $20-50$ & $5.5-85$ \\
\hline $\mathrm{C}$ & $1.27-3.81$ & $1.98-19.8$ & $1.8-18$ & $1-7.4$ & $1-5$ y $5-20$ & $1.8-38.5$ \\
\hline D & $0.00-1.27$ & $\leq 1.98$ & $\leq 1.8$ & $\leq 0.68$ & $\leq 1$ & $5.5-19.5$ \\
\hline
\end{tabular}

También a partir de la textura de un suelo puede llegar a conocerse de forma orientativa el tipo al que corresponde, mediante alguno de los distintos diagramas propuestos (Témez, 1987; MOPU, 1990; MOPT, 1992; Rawls et al., 1993; Ferrer-Julià, 2003) (véanse la tabla 7 y la fig. 10). No obstante, además de la textura es necesario tener en cuenta la profundidad a la que se encuentre una hipotética roca madre u horizonte imper- 
Figura 9. Diagrama textural para la determinación de la conductividad hidráulica a saturación $\left(K_{\text {sat }}\right.$ en $\left.\mathrm{cm} \cdot \mathrm{h}^{-1}\right)$ (López Cadenas, 1998). Los datos texturales de entrada deben ser los del horizonte menos permeable.

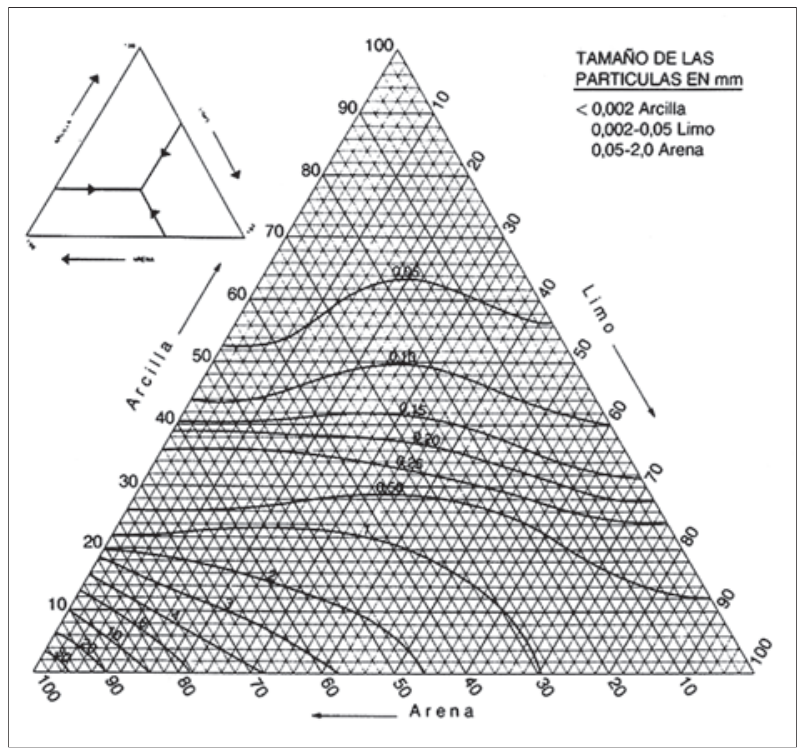

meable, o el nivel freático, de tal manera que suelos someros, suelos con un horizonte arcilloso ( $>40 \%$ de arcilla) o con un nivel freático a $60 \mathrm{~cm}$ o menos de profundidad se comportarán como suelos tipo D (NRCS, 2007), mientras que suelos profundos sobre roca madre impermeable se considerarán como tipo B.

Tabla 7. Equivalencias entre las clases texturales del USDA y los grupos hidrológicos.

\begin{tabular}{|l|c|c|c|c|c|c|}
\hline TEXTURA & $\begin{array}{c}\text { Brakensiek } \\
\text { \& Rawls } \\
(\mathbf{1 9 8 3})^{1}\end{array}$ & $\begin{array}{c}\text { Témez } \\
(\mathbf{1 9 8 7})\end{array}$ & $\begin{array}{c}\text { MOPU } \\
(\mathbf{1 9 9 0})\end{array}$ & $\begin{array}{c}\text { MOPT } \\
\mathbf{( 1 9 9 2 )}\end{array}$ & $\begin{array}{c}\text { Ferrer- } \\
\text { Julià } \\
(\mathbf{2 0 0 3})\end{array}$ & $\begin{array}{c}\text { NRCS } \\
(\mathbf{2 0 0 7})^{2}\end{array}$ \\
\hline Arenosa (Ar) & $\mathrm{A}$ & $\mathrm{A}$ & $\mathrm{A}$ & $\mathrm{A}$ & $\mathrm{A}$ & $\mathrm{A}$ \\
\hline Arenoso-franca (Ar-F) & $\mathrm{A}$ & $\mathrm{A}$ & $\mathrm{A}$ & $\mathrm{A}-\mathrm{B}$ & $\mathrm{A}$ & $\mathrm{A}^{3}-\mathrm{B}$ \\
\hline Arcillosa (a) & $\mathrm{D}$ & $\mathrm{D}$ & $\mathrm{D}$ & $\mathrm{D}$ & $\mathrm{D}$ & $\mathrm{C}^{3}-\mathrm{D}$ \\
\hline Arcillo-arenosa (a-Ar) & $\mathrm{D}$ & $\mathrm{C}$ & $\mathrm{C}$ & $\mathrm{C}$ & $\mathrm{C}$ & $\mathrm{C}^{3}$ \\
\hline Arcillo-limosa (a-L) & $\mathrm{D}$ & $\mathrm{D}$ & $\mathrm{D}$ & $\mathrm{C}$ & $\mathrm{D}$ & $\mathrm{C}^{3}$ \\
\hline Franca (F) & $\mathrm{B}$ & $\mathrm{B}$ & $\mathrm{B}$ & $\mathrm{C}$ & $\mathrm{C}$ & $\mathrm{A}^{3}-\mathrm{B}^{3}-\mathrm{C}$ \\
\hline Franco-arenosa (F-Ar) & $\mathrm{A}$ & $\mathrm{B}$ & $\mathrm{B}$ & $\mathrm{B}$ & $\mathrm{B}$ & $\mathrm{A}^{3}-\mathrm{B}$ \\
\hline Franco-arcillosa (F-a) & $\mathrm{D}$ & $\mathrm{B}-\mathrm{C}$ & $\mathrm{C}$ & $\mathrm{C}$ & $\mathrm{C}$ & $\mathrm{C}$ \\
\hline Franco-arcillo-arenosa (F-a-Ar) & $\mathrm{C}$ & $\mathrm{C}$ & $\mathrm{B}$ & $\mathrm{C}$ & $\mathrm{C}$ & $\mathrm{B}^{3}-\mathrm{C}$ \\
\hline Franco-arcillo-limosa (F-a-L) & $\mathrm{D}$ & $\mathrm{D}$ & $\mathrm{C}$ & $\mathrm{C}$ & $\mathrm{C}$ & $\mathrm{C}$ \\
\hline Franco-limosa (F-L) & $\mathrm{B}$ & $\mathrm{B}$ & $\mathrm{B}$ & $\mathrm{B}$ & $\mathrm{C}$ & $\mathrm{A}^{3}-\mathrm{B}^{3}-\mathrm{C}$ \\
\hline Limosa (L) & $\mathrm{B}$ & $\mathrm{C}$ & $\mathrm{C}$ & $\mathrm{A}$ & $\mathrm{D}$ & $\mathrm{B}^{3}$ \\
\hline
\end{tabular}

${ }^{1}$ También en Rawls et al. (1993).

${ }^{2}$ Además de la más actualizada, es la establecida oficialmente por el NRCS del USDA.

${ }^{3}$ Estructura con buenos agregados, baja densidad aparente y/o más del $35 \%$ de fragmentos rocosos. 

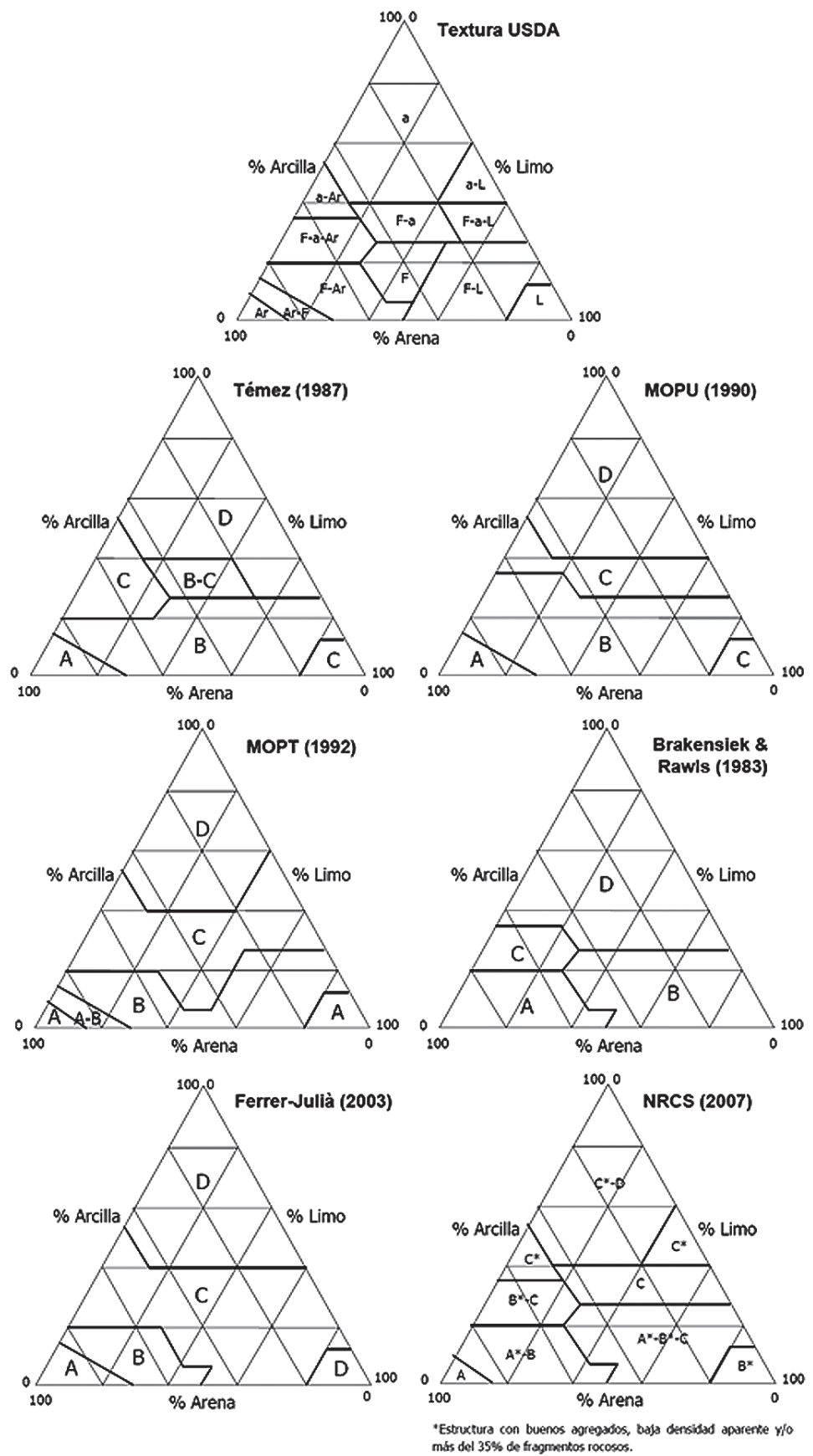

Figura 10. Diagrama textural del USDA, y diagramas para la determinación del tipo de suelo para el método del Número de Curva; elaboración propia a partir de Témez (1987), MOPU (1990), MOPT (1992), Rawls et al. (1993), Ferrer-Julià (2003) y NRCS (2007). 
En la tabla 8 se muestra la clasificación de los suelos en los diferentes grupos hidrológicos. Puede comprobarse que esta asignación es muy confusa, ya que se obtienen resultados diferentes dependiendo del criterio utilizado. Esto genera una enorme incertidumbre al determinar el tipo de suelo y el número de curva.

Tabla 8. Grupo hidrológico de los suelos objeto de estudio, según los criterios de Musgrave (1955), SSDS (1993), NRCS (1993), Nearing et al. (1996), Porta et al. (1999) y Trueba et al. (2000), Brakensiek \& Rawls (1983), Témez (1987), MOPU (1990), MOPT (1992), Ferrer-Julià (2003), NRCS (2007) y Bouwer (1969) (en Neitsch et al., 2002).

\begin{tabular}{|c|c|c|c|c|}
\hline \multirow{2}{*}{ CRITERIO } & \multirow{2}{*}{ REFERENCIA } & \multicolumn{3}{|c|}{ PM } \\
\hline & & 1 & 2 & 3 \\
\hline$f_{c}$ & Musgrave $(1955)^{1}$ & A & A & $\mathrm{A}$ \\
\hline $\mathrm{f}_{\mathrm{c}}$ & Musgrave $(1955)^{2}$ & $\mathrm{~B}$ & $\mathrm{C}$ & $\mathrm{D}$ \\
\hline$f_{c}$ & NRCS $(2007)^{1}$ & A & $\mathrm{C}$ & $\mathrm{C}$ \\
\hline $\mathrm{K}_{\text {sat }}$ & SSDS (1993) & $\mathrm{C}$ & $\mathrm{C}$ & $\mathrm{D}$ \\
\hline $\mathrm{K}_{\text {sat }}$ & NRCS (1993) & $\mathrm{C}$ & $\mathrm{C}$ & $\mathrm{D}$ \\
\hline $\mathrm{K}_{\text {sat }}$ & Nearing et al. (1996) & B & $\mathrm{C}$ & C-D \\
\hline $\mathrm{K}_{\text {sat }}$ & Porta et al. (1999) y Trueba et al. (2000) & $\mathrm{C}$ & $\mathrm{C}$ & $\mathrm{D}$ \\
\hline Clase textura & Brakensiek \& Rawls (1983) & $\mathrm{B}$ & $\mathrm{D}$ & $\mathrm{D}$ \\
\hline Clase textura & Témez (1987) & B-C & $\mathrm{B}-\mathrm{C}$ & $\mathrm{D}$ \\
\hline Clase textura & MOPU (1990) & $\mathrm{B}$ & $\mathrm{C}$ & $\mathrm{D}$ \\
\hline Clase textura & MOPT (1992) & $\mathrm{C}$ & $\mathrm{C}$ & $\mathrm{D}$ \\
\hline Clase textura & Ferrer-Julià (2003) & $\mathrm{C}$ & $\mathrm{C}$ & $\mathrm{D}$ \\
\hline Clase textura & NRCS (2007) & $\mathrm{C}$ & $\mathrm{C}$ & $\mathrm{D}$ \\
\hline$\%$ Arena & Bouwer (1969) (en Neitsch et al., 2002) & B & $\mathrm{B}-\mathrm{C}$ & $\mathrm{C}-\mathrm{D}$ \\
\hline
\end{tabular}

PM: Punto de muestreo

${ }^{1}$ Utilizando $f_{c}$ obtenida mediante el ensayo de infiltración.

${ }^{2}$ Utilizando $\mathrm{f}_{\mathrm{c}}$ obtenida a partir de la textura y la fórmula de Bouwer (1966).

No obstante, se puede decir que hay coincidencia en los resultados obtenidos con los diferentes métodos contemplados en 6 casos sobre 14 (42.9\%). Así, en el suelo de bosque (punto de muestreo 1): $\mathrm{A}=14.2 \% ; \mathrm{B}=35.7 \% ; \mathrm{C}=42.9 \% ; \mathrm{D}=0 \%$; en el suelo de matorral (punto 2): $\mathrm{A}=7.1 \% ; \mathrm{B}=7.1 \% ; \mathrm{C}=71.4 \% ; \mathrm{D}=7.1 \%$; y en el suelo de matorral del punto $3: \mathrm{A}=7.1 \% ; \mathrm{B}=0.0 \% ; \mathrm{C}=14.2 \% ; \mathrm{D}=71.4 \%$. Se puede concluir que la solución más lógica es la $\mathrm{C}-\mathrm{C}-\mathrm{D}$, respectivamente para los tres puntos de muestreo, con un $42.9 \%$ de coincidencia. De manera independiente, la situación más probable del suelo de bosque (punto 1) es $\mathrm{C}$, con un $42.9 \%$, seguida de B con un $35.7 \%$; o sea que podría calificarse como $\mathrm{C}(\mathrm{B})$; en el punto 2 (matorral) el suelo es $\mathrm{C}$, con una probabilidad del 71.4\%; y en el punto 3 (matorral), el suelo es D con un $71.4 \%$. En consecuencia, nues- 
tra propuesta sería: $\mathrm{C}(\mathrm{B}), \mathrm{C}, \mathrm{D}$, respectivamente para los tres puntos de muestreo, por reunir probabilidades superiores al $70 \%$.

Aunque es discutible la asignación final en términos de probabilidad, en nuestra opinión, en trabajos de simulación hidrológica para la estimación de avenidas, que influyen en la delimitación de zonas inundables con determinado riesgo y peligro o en el diseño de infraestructuras fluviales que deben atender a criterios específicos de seguridad, la convergencia de las clasificaciones ofrecen un mayor grado de fiabilidad para la toma de decisiones en proyectos. Hay que destacar que la estimación de caudales extraordinarios casi siempre se basa en su relación a la probabilidad de ocurrencia, y esta depende de un adecuado estudio de las precipitaciones que los generan así como de una estimación del comportamiento de los suelos de la cuenca en su transformación a escorrentías. El consenso en este caso, unido a la experiencia, resulta vital para tomar decisiones apropiadas acordes a la intensidad de los fenómenos esperados. El método del Número de Curva permite una aproximación a la realidad suficientemente buena en tiempo y forma si se restringe la variabilidad en la clasificación de los grupos hidrológicos de suelos a partir de su comportamiento en relación con la infiltración. Hawkins et al. (2009), en una reciente revisión sobre el Número de Curva, indican que existe una cierta variabilidad en \pm 1 clase, sobre todo en los grupos B y C, a partir de un intento de clasificación exhaustiva de los suelos estadounidenses en comparación con la asignación rutinaria de ciertos organismos (Hjelmfelt et al., 2001); ello nos obliga a buscar criterios de consenso ante la carencia de resultados que relacionen precipitaciones y escorrentías en la zona de estudio.

\section{Conclusiones}

En los tres puntos de muestreo analizados, ante un material geológico de partida similar, la mejora de los suelos y de sus condiciones hidrológicas es patente en el terreno arbolado (con pinar de Pinus halepensis) en comparación con los desarbolados (matorrales). Las tasas de infiltración, iniciales y finales, presentan amplias diferencias entre el pinar de repoblación y las dos zonas de matorral. No obstante, es necesario un mayor número de ensayos de infiltración y un análisis estadístico multifactorial para poder obtener resultados concluyentes.

Los suelos estudiados, tras aplicar los diferentes criterios existentes, se han clasificado en los grupos hidrológicos C (puntos de muestreo 1 y 2, con texturas franca y franco-arcillosa, respectivamente), con tendencia hacia el grupo $\mathrm{B}$ en el bosque de repoblación (punto de muestreo 1), y D (punto de muestreo 3, con textura arcillosa). Esta asignación puede servir de referencia para la determinación del número de curva en los complejos hidrológicos de estas laderas de la zona central de la cuenca del Duero. No obstante, a pesar de los diferentes intentos de delimitar los grupos de suelo para el modelo del Número de Curva, su determinación sigue presentando demasiada incertidumbre, como se ha demostrado en este trabajo. Lo ideal, a nivel técnico, para utilizar el método del Número de Curva sería consensuar unos diagramas texturales de uso sencillo, pero basados en un número suficiente de ensayos de infiltración adaptados a los suelos espa- 
ñoles. Estos diagramas disminuirían en gran medida la incertidumbre que acompaña la modelización hidrológica aplicada al diseño de obras hidráulicas, la estimación de las llanuras de inundación y el manejo del territorio en el espacio fluvial.

\section{Referencias bibliográficas}

Alaoui, A., Caduff, U., Gerke, H. H., Weingartner, R. (2011). Preferential flow effects on infiltration and runoff in grassland and forest soils. Vadose Zone Journal, 10 (1): 367-377.

Al-Azawi, S. A. (1985). Experimental evaluation of infiltration models. Journal of Hydrology (NZ), 24 (2): 77-88.

Bens, O., WAHL, N. A., Fischer, H., Huttl, R. F. (2007). Water infiltration and hydraulic conductivity in sandy cambisols: impacts of forest transformation on soil hydrological properties. European Journal of Forest Research, 126 (1): 101-109.

Blanco, R. (1999). El infiltrómetro de cilindro simple como método de cálculo de la conductividad hidráulica de los suelos. Experiencias de campo en ámbitos de montaña mediterránea. Baetica. Estudios de Arte. Geografía e Historia, 21: 9-33.

Bonell, M., Purandara, B. K., Venkatesh, B., Krishnaswamy, J., Acharya H. A. K., Singh, U. V., JAYAKUMAR, R., Chappell, N. (2010). The impact of forest use and reforestation on soil hydraulic conductivity in the Western Ghats of India: implications for surface and sub-surface hydrology. Journal of Hydrology, 391(1-2): 49-64.

Bouwer, H. (1966). Rapid field measurement of air-entry value and hydraulic conductivity of soil as significant parameters in flow system analysis. Water Resources Research, 2: 729-738.

BrakensieK, D. L., OsBorn, K. B., RAWLs, W. J. (1979a). Field manual for research in agricultural Hydrology. Agriculture Handbook, 224.

BRAKENSIEK, D. L., RAWLS, W. J. (1983). Green-Ampt infiltration model parameters for hydrologic classification of soils. En: Advances in irrigation and drainage, surviving external pressures (Borelli, J., Hasfurther, V., Burman, D., Eds.). Proceedings of American Society of Civil Engineers specialty conference (Jackson, WY). Amer. Soc. Civ., New York.

BraKensieK, D. L., RaWls, W. J., HAMON, W. R. (1979b). Aplication of an infiltrometer system for describing infiltration into soils. Transactions of the ASAE, 22: 320-333.

Buczko, U., Bens, O., Huttl, R. F. (2006). Water infiltration and hydrophobicity in forest soils of a pine-beech transformation chronosequence. Journal of Hydrology, 331(3-4): 383-395.

CammeraAt, E. L. H., Cerdà, A., Imeson, A. C. (2010). Ecohydrological adaptation of soils following land abandonment in a semi-arid environment. Ecohydrol. DOI: 10.1002/eco.161. 
Ceballos, A., Martínez-Fernández, J., Santos, F., Alonso, P. (2002). Soil-water behaviour of sandy soils under semi-arid conditions in the Duero Basin (Spain). Journal of Arid Environments, 51: 501-519

CERDÀ, A. (1995). Factores y variaciones espacio-temporales de la infiltración en los ecosistemas mediterráneos. Monografías científicas $\mathrm{n}^{\circ}$ 5. Geoforma Ediciones, Logroño.

Cerdè, A. (1997). Seasonal changes of the infiltration rates in a Mediterranean scrubland on limestone. Journal of Hydrology, 198: 209-225.

CERDÀ, A. (1998). Changes in overland flow and infiltration after a rangeland fire in a Mediterranean scrubland. Hydrol. Process., 12: 1031-1042.

CERDÀ, A. (2001). Effects of rock fragment cover on soil infiltration, interrill runoff and erosion. European Journal of Soil Science, 52: 59-68.

Cerdè, A., Doerr, S. H. (2007). Soil wettability, runoff and erodibility of major dryMediterranean land use types on calcareous soils. Hydrol. Process., 21: 2325-2336.

Cerdà, A., Imeson, A. C., Calvo, A. (1995). Fire and aspect induced differences on the erodibility and hydrology of soils at La Costera, Valencia, southeast Spain. Catena, 24: 289-304.

Chang, M. (2006). Forest Hydrology. An introduction to water and forests. Taylor and Francis Group, Boca Ratón.

Clarke, M. A., Walsh, R. P. D. (2007). A portable rainfall simulator for field assessment of splash and slopewash in remote locations. Earth Surf. Process. Landforms, 32: 2052-2069.

Collis-George, N. (1977). Infiltration equation for simple soils systems. Water Resources Research, 13: 395-403.

FAO (2006). World Reference Base for soil resources 2006. A framework for international classification, correlation and communication. World Soil Resources Report 103. Food and Agriculture Organization of the United Nations, Rome.

FERNÁNDEZ DE VillaRÁn, R. (2006). Mejora de los parámetros de cálculo del modelo del número de curva y su validación mediante un modelo hidrológico distribuido. Tesis doctoral. Universidad de Huelva, Huelva.

FERRER-Julià, M. (2003). Análisis de nuevas fuentes de datos para la estimación del parámetro número de curva: perfiles de suelos y teledetección. Cuadernos de Investigación. CEDEX, Madrid.

FitzGerald, P. D., Cossens, G. G., Rickard, D. S . (1971). Infiltration and soil physical properties. Journal of Hydrology (NZ), 10 (2): 120-126.

Green, W. H., Аmpt, C. A. (1911). Studies on soil physics, I. Flow of air and water through soils. Journal of Agricultural Sciences, 4: 1-24. 
HaAn, C. J., Johnson, H. P., Brakensiek, D. L. (1982). Hydrologic modeling of small watersheds. American Society of Agricultural Engineers, pp. 139-155.

HaRveY, A. M. (1982). The role of piping in the development of badlands and gully systems in south-east Spain. En: Badland geomorphology and piping (Bryan, R., Yair, A., Eds.). University Press, Cambridge.

Hawkins, R. H., Ward, T., Woodward, D. E., Van Mullem, J. A. (2009). Curve Number Hydrology. State of the practice. ASCE, Reston.

Hewlett, J. D. (1982). Principles of forest Hydrology. The University of Georgia Press, Athens.

HiLlel, D. (1971). Soil and water: physical principles and processes. Academic Press, New York.

HiLlel, D. (1980). Fundamentals of soil physics. Academic Press, London.

Huelmfelt, A. T., Woodward, D. A., Conaway, G., Quan, Q. D., Van Mullem, J., Hawkins, R. H. (2001). Curve Numbers, Recent Developments. XXIX IAHR Congress Proceedings, Beijing, China.

Holtan, H. N. (1961). A concept of infiltration estimates in watershed engineering. Agricultural Research Service Paper, pp. 41-51, Washington.

Horton, R. E. (1937). Determination of infiltration capacity for large drainage basins. Trans. Am. Geophys. Union, 18: 371-385.

HoRTON, R. E. (1940). An approach toward a physical interpretation of infiltration capacity. Soil Science Society of America Proceedings, 5: 399-417.

HuAng, Z. G., Ouyang, Z. Y., Li, F. R., Zheng, H., WAng, X. K. (2010). Response of runoff and soil loss to reforestation and rainfall type in red soil region of southern China. Journal of Environmental Sciences-China, 22 (11): 1765-1773.

Ilstedt, U., Malmer, A., Elke, V., Murdiyarso, D. (2007). The effect of afforestation on water infiltration in the tropics: a systematic review and meta-analysis. Forest Ecology and Management, 251 (1-2): 45-51.

LóPez CADENAS, F. (dir.) (1998). Restauración hidrológico forestal de cuencas y control de la erosión. Ed. Mundi-Prensa, Tragsa, Tragsatec, Madrid.

López Cadenas, F., Mintegui, J. A. (1986). Hidrología de superficie. Tomo I. Fundación Conde del Valle de Salazar, Madrid.

Madrid, A., Fernald, A. G., BaKer, T. T., Van Leeuwen, D. M. (2006). Evaluation of silvicultural treatment effects on infiltration, runoff, sediment yield, and soil moisture in a mixed conifer New México forest. Journal of Soil and Water Conservation, 61 (3): 159-168.

Martínez de Azagra, A., Navarro, J. (1996). Hidrología forestal. El ciclo hidrológico. Servicio de Publicaciones de la Universidad de Valladolid, Valladolid. 
Martínez de Azagra, A., Pando, V., Navarro, J., Del Río, J. (2006a). Aproximación al conocimiento de la infiltración a través del análisis dimensional. Ecología, 20: 453-470.

Martínez de Azagra, A., Pando, V., Navarro, J., Del Río, J. (2006b). A proposal of an infiltration function with ecological meaning. International Meeting Managing Forest Ecosystems: The Challenges of Climate Change. IUFRO.

MolinA, D. M. (1993). Efectos del fuego controlado en la velocidad de infiltración del agua en suelos forestales: un caso de estudio en la costa norte de California. Invest. Agrar. Sist. Recur. For., 2 (2): 173-184.

MOPT (1992). Guía para la elaboración de estudios del medio físico. Contenido y metodología. Ministerio de Obras Públicas y Transportes, Madrid.

MOPU (1990). Instrucción 5.2-I.C. Drenaje superficial. Dirección General de Carreteras. Ministerio de Obras Públicas y Urbanismo, Madrid.

Musgrave, G. W. (1955). How much rain enters the soil. Water: the yearbook of agriculture 1955.

Nearing, M. A., Liu, B., Risse, L. M., Zhang, X. (1996). Curve numbers and Green Ampt effective hydraulic conductivities. Water Resources Bulletin, 32 (1): 125-136.

Neitsch, S. L., Arnold, J. G., Kiniry, J. R., Williams, J. R., King, K. W . (2002). Soil and water assessment tool. Theoretical documentation. Texas Water Resources Institute, Texas.

NRCS (1993). Soil survey manual. http://www.nhq.nrcs.usda.gov/JDV/ssmnew.

NRCS (2007). Part 630. Hydrology. National Engineering Handbook. Washington D.C.

OVERTON, D. E. (1964). Mathematical refinement of an infiltration equation for watershed engineering. USDA Agricultural Research Service, Washington.

PhiLIP, J. R. (1957). The theory of infiltration. Chapter 1. Soil Science, 83: 345-357

Porta, J., LóPez-Acevedo, M., Roquero, C. (1999). Edafología . Para la agricultura y el medio ambiente. Ed. Mundi-Prensa, Madrid.

RAVI, V., WiLliams, J. R. (1998). Estimation of infiltration rate in the vadose zone: compilation of simple mathematical models. Volume I. EPA, Ada.

Rawls, W. J., Ahuja, L. R., Brakensiek, D., Shirmohammadi, A. (1993). Infiltration and soil water movement. En: Handbook of hydrology (Maidment, D. R., ed.). Ed. McGraw Hill, New York.

RAWLS, W. L., BRAKENSIEK, D. L. (1983). A procedure to predict Green and Ampt infiltration parameters. En: Proc. Am. Soc. of Agr. Eng. on Advan. in infiltration, Chicago.

SCS (1991). Engineering field handbook. Chapter 2. SCS, Washington D.C.

SCS (1954). National Engineering Handbook, section 4 Hydrology. USDA-SCS, Washington D.C. 
SIDIRAS, N., RoTH, C. H. (1987). Infiltration measurements with double-ring infiltrometers and a rainfall simulator under different surface conditions on an oxisol. Soil and Tillage Research, 9 (2): 161-168.

SkAGGs, R. W., KhaheEl, R. (1982). Infiltration. En: Hydrologic modeling of small watersheds (Haan, C. T., Johnson, H. P., Brakensiek, D. L., eds.). ASAE Monograph No. 5, St. Joseph.

SSDS (1993). Soil survey manual. Handbook 18. Soil Survey Division Staff. Soil Conservation Service (USDA), Washington, D.C.

TÉmEZ, J. R. (1987). Cálculo hidrometeorológico de caudales máximos en pequeñas cuencas naturales. MOPU. Dirección General de Carreteras, Madrid.

Touma, J., Albergel, J. (1992). Determining soil hydrologic properties from rain simulator or double ring infiltrometer experiments: a comparison. Journal of Hydrology, 135: 73-86.

TRICKER, A. S. (1978). The infiltration cylinder: some comments on its use. Journal of Hydrology, 36: 383-391.

Trueba, C., Millán, R., Sdchimd, T., Lago, C., Gutiérrez, J. (2000). Estimación de índices de vulnerabilidad radiológica para los suelos peninsulares españoles. CIEMAT, Madrid.

VALerón, B., MeiXner, T. (2010). Overland flow generation in chaparral ecosystems: temporal and spatial variability. Hydrological Processes, 24 (1): 65-75.

Williams, J. R., Ouyang, Y., Chen, J. S., Ravi, V. (1998). Estimation of infiltration rate in the vadose zone: application of selected models. Volume II. EPA, Ada.

YAseef, N. R., YAKIR, D., Rotenberg, E., Schiller, G., Cohen, S. (2010). Ecohydrology of a semi-arid forest: partitioning among water balance components and its implications for predicted precipitation changes. Ecohydrology, 3 (2): 143-154.

Zapata, A., ManZano, F. (2008). Influence of six tree species on water infiltration. Agrociencia, 42 (7): 835-845.

Zegelin, S. J., White, I. (1982). Design for a field sprinkler infiltrometer. Soil Science Society of America Journal, 46-6: 1129-1133. 